\title{
Application and Practice of Remote Measurement and Control Platform in the Experiment Teaching Course of "Data Acquisition and Interface Technology"
}

\author{
Liang Ge, Chun Yang, Simeng Zhu, Bin Li, Mingjiang Shi, Lai Xin \\ College of Mechanical and Electronic Engineering, Southwest Petroleum University, Chengdu, China \\ Email: cgroad@swpu.edu.cn
}

How to cite this paper: Ge, L., Yang, C., Zhu, S. M., Li, B., Shi, M. J., \& Xin, L. (2018). Application and Practice of Remote Measurement and Control Platform in the Experiment Teaching Course of "Data Acquisition and Interface Technology". Creative Education, 9, 779-790.

https://doi.org/10.4236/ce.2018.95058

Received: March 26, 2018

Accepted: April 21, 2018

Published: April 24, 2018

Copyright $\odot 2018$ by authors and Scientific Research Publishing Inc. This work is licensed under the Creative Commons Attribution International License (CC BY 4.0).

http://creativecommons.org/licenses/by/4.0/

\begin{abstract}
Data Acquisition and Interface Technology is a compulsory course for the major of measurement and control technology and instrument in Southwest Petroleum University, and it is a basic course combining theory and practice closely. The traditional experiment teaching is limited by various conditions. Students can only complete the specific experiment content with limited experiment equipment in limited time and place. It is an effective way to solve the above problems by introducing virtual simulation technology to construct the remote measure and control experiment platform to carry on the reform of the traditional experiment teaching mode. The remote measurement and control platform uses virtual technology to form the test function into a software module with image-realistic panel controls, to complete the test and processing tasks as if doing experiments in the laboratory, to move the experiment teaching into the classroom, to move into the network and life, and to realize the perfect fusion of theory and practice. It has been proved that the application of the remote measurement and control platform in the experimental teaching of the course of Data Acquisition and Interface Technology can improve the teaching effect. Moreover, the cost is low and the efficiency is high, so that students cannot be limited by time and space, improve their self-learning ability and practical innovation ability, which is of great significance in the teaching of the course of measurement and control technology.
\end{abstract}

\section{Keywords}

Remote Measurement and Control Platform, Data Acquisition and Interface Technology, Experimental Teaching, Teaching Reform, Effect Evaluation 


\section{Introduction}

The experiment course is based on the theory teaching, and it is the practical teaching link that serves the course, it has the effect of finishing the theory course. In the traditional teaching mode of measurement and control technology class, the theory class is separated from the experiment class, which leads to the skills training cannot be timely theoretical guidance, and the theory class is not combined with production, life reality, so that students do not master the skills in place. The traditional experiment teaching is restricted by various conditions, and the students can only use the limited experimental equipment to complete the specific experiment content in a limited time and place. Traditional experiment teaching is only an adjunct of validating and consolidating theory teaching, so it lacks of design, research, comprehensiveness, creativity and practicability. The above problems expose the drawbacks of the experiment in the course of measurement and control technology, that is, the current experimental environment is difficult to meet the needs of teaching, and urgently needs to be improved. Under the condition that the existing experimental teaching resources are insufficient and the equipment is updated slowly, we can solve the above problems by establishing the remote measurement and control experiment platform and introducing it into the experiment teaching (Wang \& Yao, 2005; Zhong, 2005).

The introduction of the remote measurement and control experiment platform makes the knowledge of many courses in universities (measurement and control principle, SCM application technology, signal analysis, etc. (Gu \& Zhu, 2010) organically combined. By using the advantage of the remote measurement and control experiment platform, the virtual simulation teaching and project teaching are closely combined to achieve a higher level of "teaching, learning and doing" integration, which is very conducive to carry out the project-based course teaching reform based on the working process (Wang, Huang, \& Wang, 2010). With the rapid development of science and technology and the urgent need of the country for high quality talents, the introduction of the remote measurement and control platform to assist the experimental teaching of the major courses of measurement and control, bringing new changes to the experimental teaching mode, is the inevitable trend of the future teaching development, and the popularization of the remote measurement and control platform will exert far-reaching influence on the development of each science teaching field (Luo \& Zhang, 2016; Ma \& Chen, 2016).

\section{Realization of Remote Measurement and Control Experiment Platform}

The remote measurement and control experiment platform is based on the virtual simulation technology, using LabVIEW software programming to realize the virtual simulation and remote measurement and control demonstration of 
the data acquisition and interface technology knowledge in the course of measurement and control technology (Kalkman, 1995). Virtual simulation teaching refers to the use of physical and computer software to simulate the real situation, so that students in the simulation of the situation to explore and study.

\subsection{Development Environment}

LabVIEW programming software based on virtual instrument design has a unique advantage in virtual instrument design, especially suitable for the design of virtual simulation system. Compared to other development systems, LabVIEW provides intuitive user interface, flexible and efficient program design, extensive and in-depth hardware platform, easy to follow up development and porting system design (Kirkman \& Buksh, 1992).

LabVIEW is a graphical programming language that uses icons instead of text lines to create applications. Data flow programming is used. Data flow direction between nodes in a block diagram determines the execution order of a program. It uses an icon to represent the function and a line to indicate the data flow. LabVIEW's programming environment consists of three main sections: the front panel, the program block diagram, and the icon/wiring board. The front panel is the VI user interface, and the input controls and display controls are used to create front panels, which are the interactive input and output terminals of VI. The Program block diagram is a graphical source code. The front panel object is displayed as a writing terminal in the program block diagram. An icon appears in the upper-right corner of each VI's front panel window and program block diagram window. A wiring board is a set of terminals that correspond to an input control and a display control in VI, similar to a list of function call parameters in a text programming language.

\subsection{Build Process}

As shown in Figure 1, the remote measurement and control laboratory and its teaching management platform framework mainly consist of user login module, user management module, remote measurement and control experiment module and data recording module. The experimental project includes: Digital signal input module, digital signal output module, analog signal input module, analog signal output module, etc.

The operation interface of remote measurement and control platform is shown in Figure 2, which consists of 7 columns such as platform introduction, experiment content, experiment report, online answering questions, related links, relevant downloads, system help, etc. User login is divided into administrator login and student login two rights, access to the administrator interface, there are experimental management, online communication management, announcement system, related downloads and other content. The administrator can add and delete the experiment item, can answer the hot question that the student puts forward in the forum, release the experiment time, place, request 


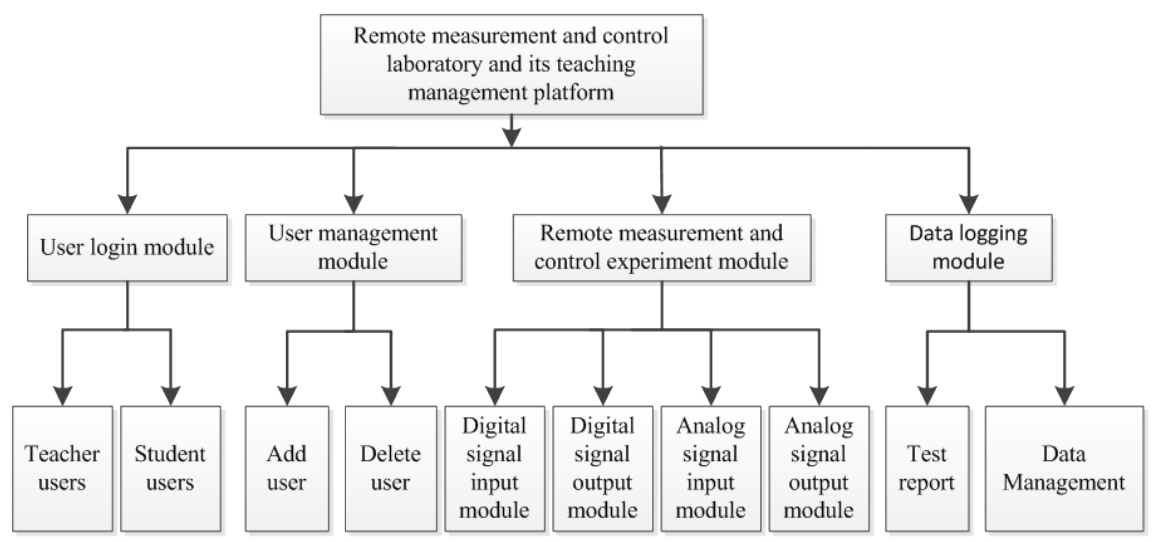

Figure 1. Block diagram of remote measurement and control laboratory and its teaching management platform.

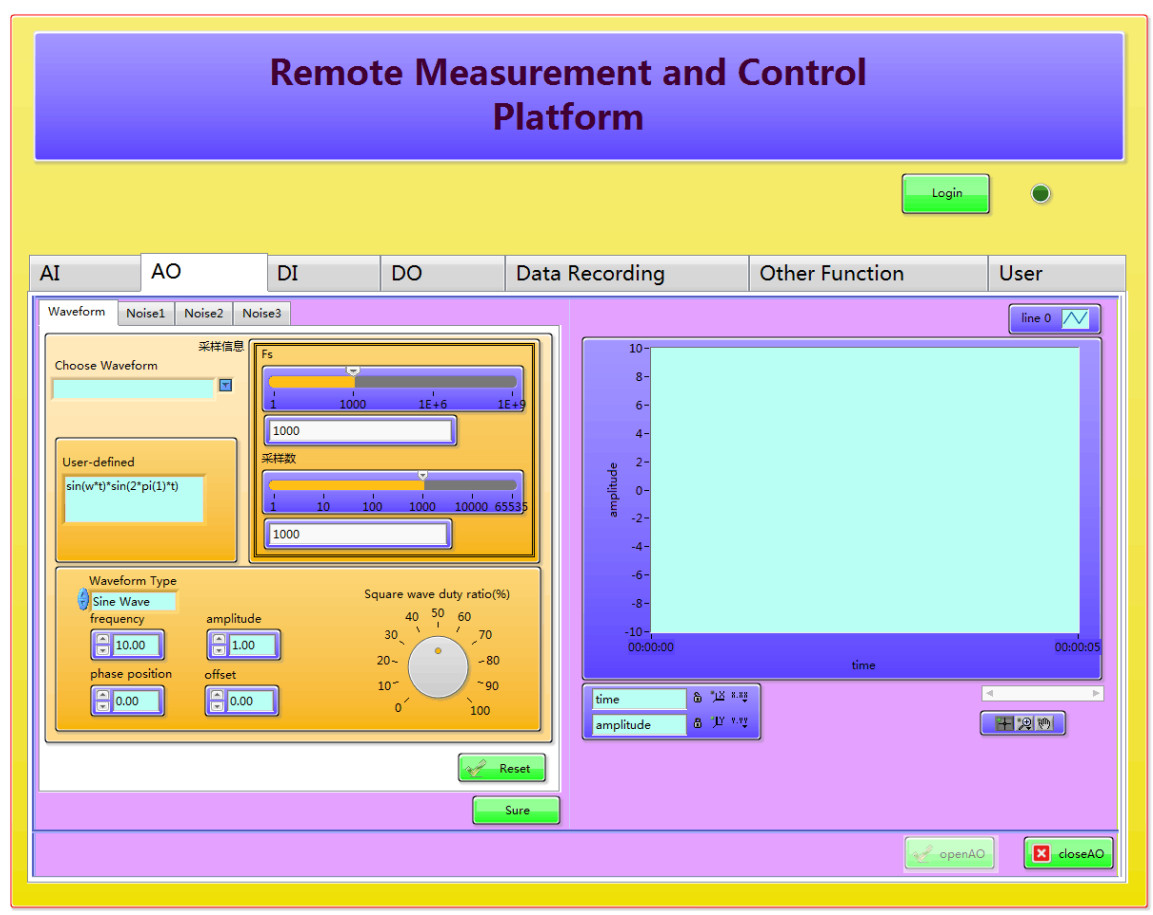

Figure 2. Operating interface of remote measurement and control experiment platform.

and so on in the bulletin column, in the related downloading can carry on the experiment content, the experiment report downloading.

\subsection{Main Features}

The remote measurement and control experimental platform provides teachers and students with an extensible, simple and easy-to-use experimental platform. Using this experimental platform, we can easily construct the related experiments in the measurement and control technology courses with rich manifestations and complete the requirements of data collection experiments. Its main features are as follows:

1) Realistic experimental environment. With the help of remote measurement 
and control experimental platform, introducing project tasks and objectives, and showing project results, students can have an intuitive understanding of the project, which can satisfy their online independent learning of data acquisition and interface technology-related experiments.

2) High performance, easy to expand. The virtual instrument developed on the basis of computer technology has its own advantages of multiple operating platform, high speed operation, large capacity storage and network communication, and the advantages of virtual instrument in this aspect will become more and more obvious with the development of computer technology and performance. The "software-defined instrument" feature of virtual instrument enables users to achieve the goal of designing products with the lowest cost and the fastest time.

3) Good versatility. By using LabVIEW's data communication functions, it can conveniently communicate with the virtual serial port of SCM to realize the analysis and processing of the data collected by the slave computer. The installability of the software makes the experiment of data acquisition and interface technology not only out of the laboratory, into the classroom, but also into the space of self-study.

4) Highly reliable experimental environment. The purpose of the remote measurement and control experiment platform is to replace the actual controlled object by the virtual simulated controlled object, and to verify the correctness of the experiment program. Therefore, an accurate remote measurement and control experiment platform is needed, otherwise the experiment may fail. The remote measurement and control platform based on virtual simulation technology can meet the reliability requirement of the experiment.

5) User-friendly and easy to operate. Using the GUI provided by LabVIEW software, we can design a set of friendly man-machine interface to realize the real-time control of the SCM measurement and control system, visualize the complex problem, operate simply and conveniently, and make it easier for students to understand.

6) Perfect Teaching Evaluation System. The establishment of a scientific and reasonable way to assess and evaluate the results of experiments, including the preparation and preparation of experiments, the process and results of experiments, the report of experiments and the completion of design-type experiments, will help to improve students' enthusiasm for experiments and achieve the expected goal of experimental courses.

\section{Application of Remote Measurement and Control Platform in Experimental Teaching of the Course of Data Acquisition and Interface Technology}

Applying the remote measurement and control platform based on virtual simulation technology to the experimental teaching of the course of Data Acquisition and Interface Technology, a new experimental teaching mode of university was 
explored with three levels of "teacher management-student virtual experiment-laboratory teaching" and four links "project introduction-making plan-implementation plan-results display and evaluation". Figure 3 is the application of the remote measurement and control platform in the experimental teaching of Data Acquisition and Interface Technology.

The following is a brief introduction to the application of the remote measurement and control platform in the experimental teaching of data acquisition and interface technology:

1) Before the experiment

In the project introduction link, the teacher needs to construct the experimental situation, carefully prepare each experiment, let the students preview in advance, basic understanding of the experimental content, the problems that may be encountered in the experiment are given in an appropriate form. According to their own specific circumstances, the students can use the remote measurement and control platform in the computer to preview the experiment before, and clear the experimental task. In the formulation of planning links, students use the vivid and intelligent remote measurement and control platform to fully grasp the principle of the experiment, understand the use of the instrument, be familiar with the experimental process and some other preparatory work before the experiment. For the design experiment, the experiment instructor first asks the student to design the experiment plan, the experiment procedure and the necessary experimental instrument before doing the experiment. The students use the remote measurement and control platform for simulation experiments, if the experimental scheme is feasible, then transmit own design plan to the teacher and the schoolmate through the network transmission tool to carry on the exchange, if they all agree that the plan is feasible, may carry on the

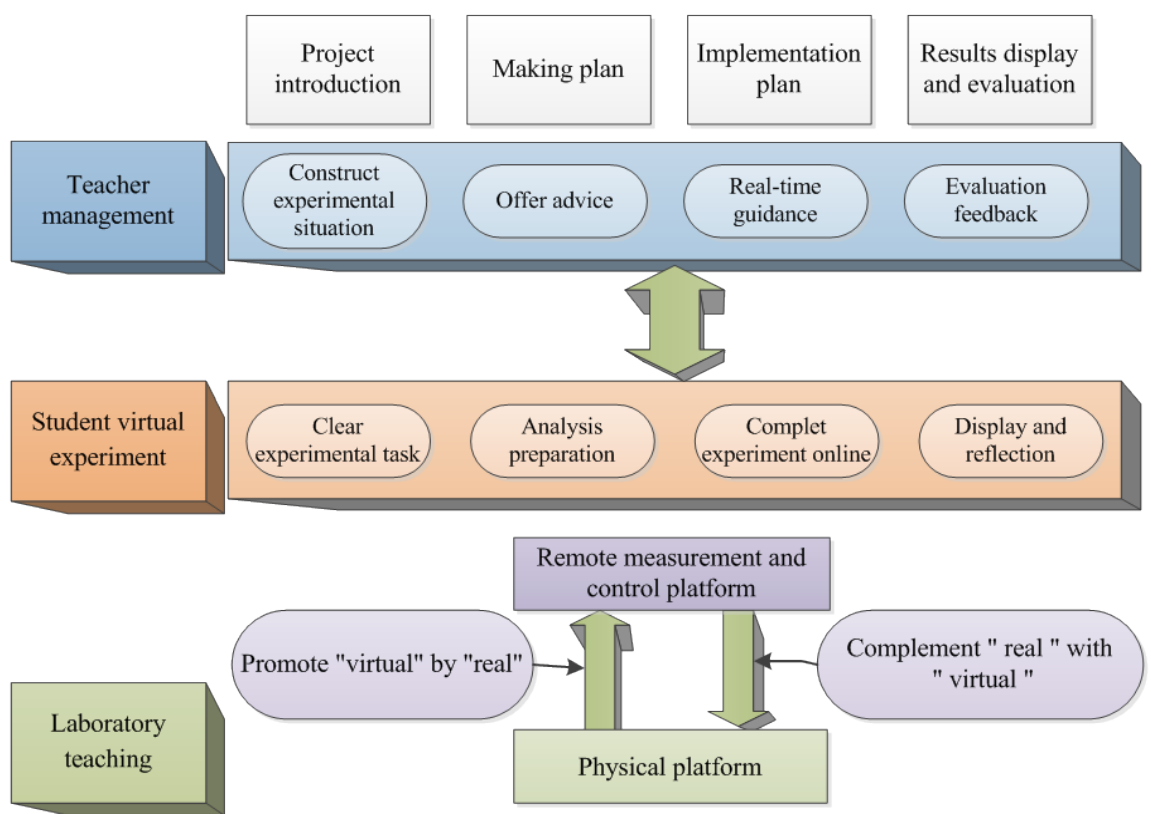

Figure 3. Application process of remote measurement and control platform. 
concrete experiment operation. Using remote measurement and control platform to communicate through network can make students avoid unnecessary loss and waste of time which caused by blind operation and greatly improves the efficiency of experiment.

2) In the process of experiment

In the implementation of the planning process, students will encounter different problems in the course of the experiment, but students can find solutions through the remote control platform to at any time on the computer. Different students' understand, accept, hands-on ability are different, the problems encountered in the experiment will be different, through the guidance and supervision of teachers, mutual cooperation between students to solve. Not only can reduce the workload of teachers, but also students to cooperate with each other, more conducive to training students to solve their own problems. Using computer to collect and process data during the experiment can avoid the error caused by human factors and improve teaching efficiency and quality. Because of the different personal abilities of the students, some students can finish the experiment operation quickly. These students can use the extra time to know whether other methods can be used to do this experiment through circuit simulation. Interested students may try other experimental methods to further study the experiment. This not only enriches the students' professional knowledge, broadens their knowledge and horizons, but also improves their curiosity and fosters their sense of innovation.

3) After the experiment

In the demonstration and evaluation link, students can use the network or the local area network to transmit the experiment report to the teacher. On the net, the teacher can quickly check the concrete situation of the students' experiment report and feedback the problems in the experiment report to the students in time. In this way, the problems that students encounter in the experiment are solved in time, which shortens the time of communication between teachers and students, improves the teaching efficiency of the experiment, and deepens the students' understanding and consolidation of the experiment. In the past, whenever students want to review the experiment done, because the time interval is too long to forget the specific circumstances when the experiment was done, can not achieve the effect of review, and the school can not provide students with access to the laboratory to review the experiment done at any time because of the equipment and time. However, this problem can be solved well by remote measurement and control platform. If the students need to review can be based on their own time and anywhere through the remote measurement and control platform, and simulation experiments can truly reproduce the experimental process and experimental phenomena, can help students recall the details of the experiment at that time, and further deepen the students understanding of the experiment and understanding.

Applying the remote measurement and control platform to the experimental 
teaching, the physical platform of the laboratory promotes "virtual" by "real" and extracts the virtual experiment from the physical platform ; The online remote measurement and control platform can complement "real" with "virtual "and improve the experimental teaching platform with the combination of virtual and reality, .

\section{Application of Remote Measurement and Control Platform in Experimental Teaching of Data Acquisition and Interface Technology}

Under the remote measurement and control platform, using circuit simulation to assist experimental teaching can give full play to students' initiative and creativity, fully display the students' innovative spirit in the experiments, and enable students to have more opportunities to apply the knowledge they have learned to the experiments under different circumstances, thus promoting students' overall mastery of the relevant knowledge of measurement and control specialty and improving their ability to truly solve practical problems.

Figure 4 is a comparative analysis of the students' actual operation time and experiment completion before and after applying the remote measurement and control platform. By using the remote measurement and control platform, students can easily master the precautions, experimental principles and use of instruments before doing experiments. Experimental teachers no longer spend as much energy and time as they did in the past such as giving students pre-experiment preparations and emphasizing the precautions. After a vivid and realistic preview before the experiment, the students will do well when they finish the experiment independently in the lab. After using a remote measurement and control platform for students to enter the practical laboratory experiment and test completion time for recording, compared with traditional laboratory experiments found that the preview of using the remote measurement and control platform is much better than the preview of the traditional experiment class. At the same time, a random sampling survey of the students in Southwest Petroleum University showed that more than $60 \%$ of the students had great interest in introducing online remote experimental platform to assist learning, and were willing to devote their time and energy to this activity.
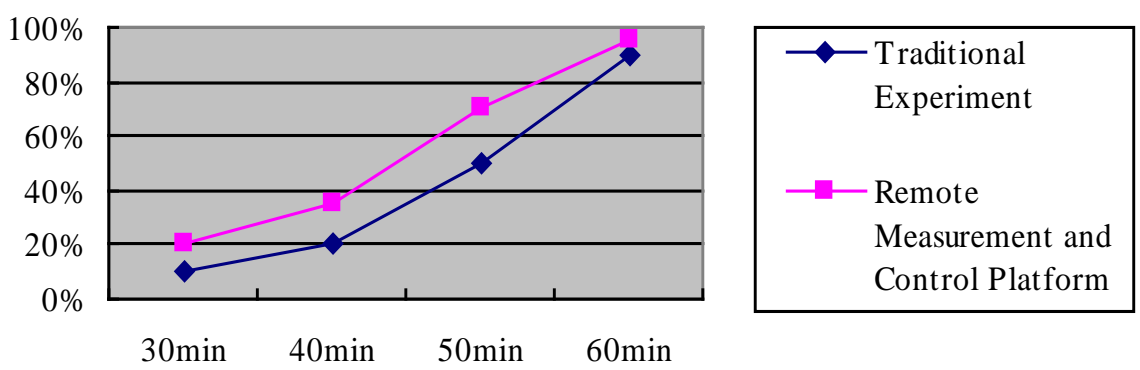

Figure 4. Analysis of Experiment Time and Completion Situation before and after Applying Remote Measurement and Control Platform. 
Figure 5 is a questionnaire about the damage of laboratory equipment and instruments before and after the application of remote measurement and control platform. As can be seen from the data in the table, the damage rate of laboratory equipment and instruments is reduced after the application of remote measurement and control platform, which effectively reduces the investment of laboratory equipment funds and has obvious economic advantages. At the same time, the remote measurement and control experimental platform can transmit data through the Network; the instructor can monitor the experimental process through the computer, and can manage dozens or even hundreds of students to do the experiment at the same time. On the basis of not adding too much funds, the experimental conditions have been improved, the experimental teaching methods and experimental means have been perfected, and the training of students' practical ability has been achieved.

Figure 6 is a comparison between the distribution areas of the test scores of the experimental courses before and after the application of the remote measurement and control platform to the students of 2 classes of the measurement and control major. Through the investigation, many students have encountered the time conflict, the physical factors and other unexpected events in the experiment class, which affect the normal course of the experimental courses. However, the traditional experimental courses in the fixed time of the laboratory, students can only make a choice, can not be balanced. After the introduction of remote measurement and control platform, students can master the initiative of learning, not limited by time and space, selective in the appropriate time to use the computer remote measurement and control platform for autonomous learning.

It can be seen that after the introduction of the remote measurement and control platform, the students' mastery of the teaching content of the experiment course becomes better, and the test result of the experiment is improved obviously.

Figure 7 is the investigation results of the students' preview, review and extended learning using the remote measurement and control platform. The remote measurement and control platform adopts the experimental mode of combining virtual simulation software and physical verification, and simulates the real situation by using simple physical object and computer software, so that
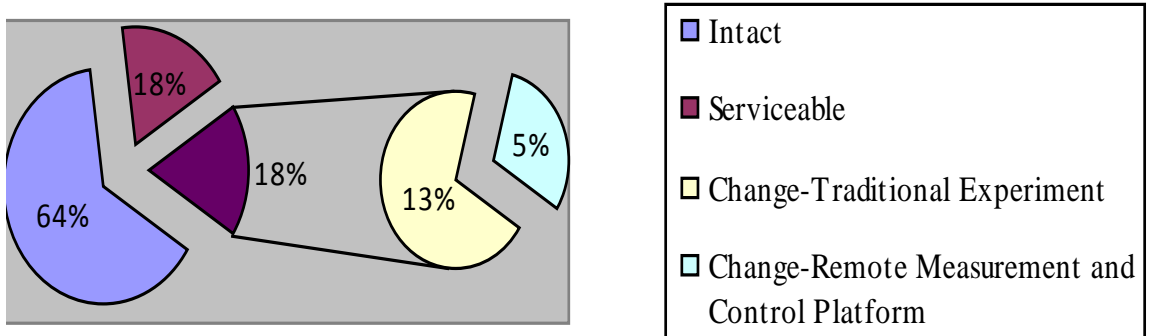

Figure 5. The damage of laboratory equipment and instruments before and after the application of remote measurement and control platform. 


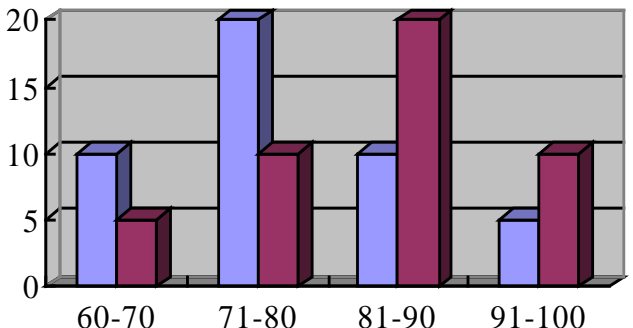

$\square$ Traditional Experiment

Remote Measurement and Control Platform

Figure 6. Comparison between the distribution areas of the test scores of the experimental courses before and after the application.

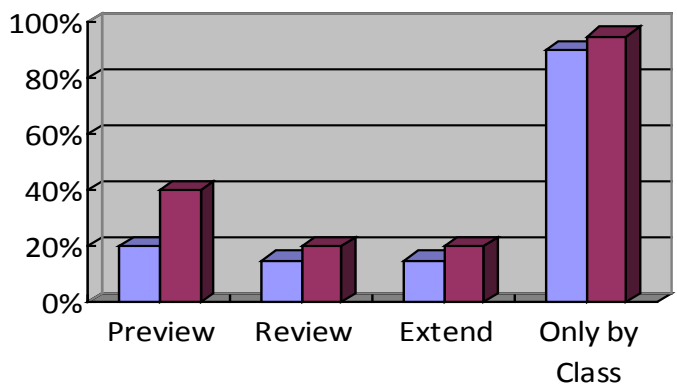

Figure 7. Students' utilization of remote measurement and control platform.

students can explore and learn in the simulated situation. This kind of vivid teaching method enables the student to enter the corresponding situation in the short time, can grasp the operation skill quicker. Students complete self-study experiment theory, instrument operation method and experiment content before class, accomplish experiment independently, cultivate the ability of independently acquiring knowledge and applying existing knowledge to open up, develop and create new knowledge. In addition to the normal course arrangement, students can also study and review according to their own situation and interest in other appropriate time, and truly realize the autonomy of learning.

It can be seen that the introduction of the remote measurement and control platform enables students to gain the autonomy of learning, greatly improve their interest in learning and stimulate their learning desire and potential, to create a helpful space to the cultivation of innovative consciousness and personality development.

\section{Summary and Prospect}

The introduction of the remote measurement and control platform into the experimental teaching of the course of Data Acquisition and Interface Technology brings new changes to the experimental teaching, which is embodied in the following aspects:

1) Improve the effect of experimental teaching. The method of introducing remote measurement and control platform into the experimental teaching of Data Acquisition and Interface Technology is vivid and close to the real scene, which is helpful to raise students' interest, make students enter the correspond- 
ing situation in a short time, and make the real experience operate in reality. While significantly improving teaching conditions and improving teaching effect, students can use their own computers to conduct simulation preview, deepen the grasp of experimental operation and theoretical knowledge, not only improve the efficiency of doing real experiments, but also benefit the teaching and practice of experimental courses of measurement and control technology.

2) Effectively reduce the investment of experimental equipment funds. The remote measurement and control platform has little hardware input and no equipment maintenance, so the economic advantage is obvious. It can not only make up for the lack of experimental equipment and components, but also eliminate the raw material consumption and equipment damage factors, can help better carry out teaching activities.

3) Break the time and space limit and provide students with unlimited practical opportunities. The remote measurement and control platform has good practicability in experiment teaching and some experiments which are not suitable for laboratory operation. By using the remote measurement and control platform to carry out experiments, students can go beyond the limits of time and space and make modifications and debugs independently, especially for complicated experiments and comprehensive experiments that are difficult to complete in traditional teaching.

4) Improve students' innovative ability and promote students' independent learning. Remote measurement and control platform can use the advantages of computer software to create a flexible and diverse work scene, not only to cultivate the ability of students' self-study, and fully tap the creative potential of students, improve their interest in doing experiments, and is of great help to train students' personality development and innovative ability.

The introduction of remote measurement and control platform in experimental teaching of Data Acquisition and Interface Technology can not only solve the drawbacks existing in traditional teaching, but also improve teaching efficiency and quality, at the same time reduce teachers' work burden and improve students' interest in learning measurement and control technology courses, broaden students' knowledge horizons and train students' innovative ability. The virtual simulation function of remote measurement and control platform can vividly show and simulate some experiments and phenomena that can not be realized under the existing experimental environment, so as to benefit the students' learning. The remote measurement and control platform based on virtual simulation technology has the characteristics of interactivity, openness and innovation, which will lead the current world science and technology development trend into undergraduate course teaching, so that students can reach the newest science and technology field and apply their knowledge.

\section{Fund}

This work is supported by Self research project of equipment in Southwest Petroleum University and the University student innovation industry project (No. 
201610615013).

\section{References}

Gu, N., \& Zhu, L. (2010). Construction of the Virtual MCU Experimental Simulation Platform. Experimental Technology and Management, 10, 105-107.

Kalkman, C. J. (1995). LabVIEW: A Software System for Data Acquisition, Data Analysis, and Instrument Control. Journal of Clinical Monitoring, 11, 51-58.

Kirkman, I. W., \& Buksh, P. A. (1992). Data Acquisition and Control Using National Instruments' "LabVIEW" Software. Review of Scientific Instruments, 63, 869-872.

Luo, H., \& Zhang, X. (2016). Exploration on Opening and Sharing Mode of Virtual Simulation Experimental Teaching Center. Experimental Technology \& Management, 10, 237-241.

Ma, X., \& Chen, L. (2016). Exploration on Digital Circuit Experimental Teaching Based on Virtual Simulation Technology. Experimental Technology \& Management, 10, 132-134.

Wang, B. Y., Huang, X. Y., \& Wang, D. R. (2010). Application of Virtual Simulation Platform in the Teaching of Single-Chip Microcomputer. Journal of West Anhui University, 5, 38-41.

Wang, Z. W., \& Yao, B. W. (2005). Design and Reality on a Remote Measurement and Control Platform. China Measurement Technology, 3, 71-72.

Zhong, L. (2005). Remote Measurement and Control System Based on Data Socket Technique. Instrument Standardization \& Metrology, 2, 27-29. 